\title{
ХИРУРГИЧЕСКАЯ РЕВАСКУЛЯРИЗАЦИЯ МИОКАРДА ПРИ ОСТРОМ КОРОНАРНОМ СИНДРОМЕ. ОБЗОР ЛИТЕРАТУРЫ
}

\section{SURGICAL MYOCARDIAL REVASCULARIZATION IN ACUTE CORONARY SYNDROME. REVIEW}

A. Jalilov

A. Vischipanov

Summary. A literature review on the topic of acute coronary syndrome without persistent ST segment elevation is presented. The use of this term in clinical practice makes it easier to diagnose and, as a result, accelerate the provision of medical care at the prehospital stage, after the patient enters the hospital and in the first few days of hospital treatment to determine treatment tactics. Invasive treatments for acute coronary syndrome include bypass coronary artery bypass surgery and percutaneous coronary intervention. According to current recommendations of the last decades, the trends in the active treatment of acute coronary syndrome are shifted towards endovascular procedures. However, in a number of cases, the most effective benefit is an open coronary artery bypass grafting operation. The article discusses the options currently used for surgical myocardial revascularization of patients. To date, surgical methods of treating acute coronary syndrome include: coronary artery bypass grafting in conditions of cardiopulmonary bypass in combination with various myocardial protection options, including using various methods of cardioplegia; coronary artery bypass grafting on a working heart - the so-called "off-pump" technique, and coronary artery bypass grafting under conditions of cardiopulmonary bypass perfusion. Studying the problems associated with the tasks and possibilities of surgical treatment of acute coronary syndrome will increase the duration and quality of life of patients with a cardiovascular profile.

Keywords: coronary artery bypass grafting, acute coronary syndrome, myocardial infarction, myocardial revascularization.

\author{
Жалилов Адхам Кахрамонович \\ К.м.н., Каршинский филиал республиканский \\ специализированный научно-практический \\ медицинский центр кардиологии \\ Jalilov_adham@mail.ru \\ Вищипанов Артем Сергеевич \\ Д.м.н., професссор, ФГАОУ ВО «Российский \\ Национальный Исследовательский Медицинский \\ Университет им. Н.И. Пирогова» Минздрава России
}

Аннотация. Представлен литературный обзор по теме острого коронарного синдрома без стойкого подъёма сегмента ST. Использование данного термина в клинической практике позволяет облегчить диагностику и, как следствие, ускорить оказание медицинской помощи на догоспитальном этапе, после поступления больного в стационар и в первые несколько суток стационарного лечения, для определения тактики лечения. К инвазивным методам лечения острого коронарного синдрома относят операции обходного шунтирования коронарных артерий и чрескожное коронарное вмешательство. Тенденции в области активного лечения острого коронарного синдрома, согласно актуальным рекомендациям последних десятилетий, смещены в сторону эндоваскулярных процедур. Однако, в целом ряде случаев, наиболее эффективным пособием является открытая операция аортокоронарного шунтирования. В статье рассмотрены варианты хирургической реваскуляризации миокарда, используемые в настоящее время. На сегодняшний день, к хирургическим методам лечения острого коронарного синдрома относятся: коронарное шунтирование в условиях искусственного кровообращения в сочетании с различными вариантами защиты миокарда, в том числе с помощью различных методов кардиоплегии; аортокоронарное шунтирование на работающем сердце - т.н. методика «оff-рuтр», и коронарное шунтирование в условиях искусственного кровообращения на параллельной перфузии. Изучение проблем, связанных с задачами и возможностями хирургического лечения острого коронарного синдрома, позволит увеличить продолжительность и качество жизни пациентов сердечно-сосудистого профиля.

Ключевые слова: аортокоронарное шунтирование, острый коронарный синдром, инфаркт миокарда, реваскуляризация миокарда.

на сегодняшний день являются ведущей причиной смертности в индустриально развитых странах. По данным Европейского общества кардиологов (ESC), ожидается, что к 2020 г. данная группа заболеваний займёт первое место в структуре смертности и в развивающихся странах [2]. При этом, одной из ведущих причин смертности от сердечно-сосудистой патологии является острый коронарный синдром (ОКС) $[2,3,4]$. Несмотря на современные возможности лечения, такие показатели как смертность, 
частота инфаркта миокарда (ИМ) и повторных госпитализаций у пациентов с ОКС остаются высокими [5].

Термин острый коронарный синдром (ОКС или Acute Coronary Syndrome) был введён V. Fuster и его коллегами в 1985 году для объединения различных синдромов по характеру патофизиологических процессов, противопоставляя ИМ и нестабильную стенокардию стабильной стенокардии напряжения. ОКС представляет собой обострение течения ишемической болезни сердца (ИБС), то есть любую группу клинических признаков или симптомов, позволяющих подозревать начальный период развития нестабильной стенокардии или острый ИМ. При этом диагностика ОКС у пациентов с болью в груди может быть достаточно трудной задачей, особенно при отсутствии характерных симптомов или изменений на электрокардиограмме, а именно, отсутствие подъёма сегмента ST[1]. Данная эта группа пациентов заслуживает особого внимания, поскольку позволяет начать своевременное адекватное лечение, не дожидаясь формирования развернутой клинической картины и ухудшения симптоматики $[9,11]$.

Положение сегмента ST в первые часы развития ОKC является основополагающим фактором, с точки зрения прогноза заболевания. На сегодняшний день достоверно установлено, что характер и срочность вмешательства для восстановления коронарной перфузии, во многом, определяются положением сегмента ST на ЭКГ относительно изоэлектрической линии.

Кроме того, согласно результатам крупных эпидемиологических исследований, около 50\% пациентов с подозрением на ОКС, в момент госпитализации не имеют диагностически значимых изменений ЭКГ [6]. Это больные со «старой» полной блокадой левой ножки пучка Гиса, с перенесенным ранее крупноочаговым инфарктом миокарда (ИМ), аневризмой левого желудочка (при отсутствии предшествующих ЭКГ для сравнения), больные с изменениями сегмента ST, не достигающими достаточной для постановки диагноза степени.[10]

Только 1/3 больных с ОКС описывают боль как типичную: локализованную за грудиной, имеющую характер жжения или сдавления [7]. В остальных случаях больные сообщают о дискомфорте, тяжести в груди, сильной слабости, ноющей боли слева от грудины. Иногда у ряда пациентов с ОКС, особенно часто у больных сахарным диабетом, женщин, молодых пациентов (2540 лет) и пациентов старшего возраста (старше 75 лет), отсутствует ощущение боли или боль носит нетипичный характер [8].

По мере работы с пациентом и увеличения знаний о нём, благодаря дополнительным методам исследо- вания, диагноз ОКС уточняется. В первую очередь с помощью лабораторных показателей (максимального уровня сердечного тропонина, активности ферментов) и данных визуализирующих методов. Диагноз ОКС без подъёма сегмента ST, в свою очередь, уточняется и переходит в нестабильную стенокардию, либо ИМ без зубца Q, либо в некоронарогенное поражение миокарда. Если признаки некроза миокарда обнаруживают у больного с ОКС, у которого не было начальных изменений на ЭКГ в виде стойких подъемов сегмента ST, то это состояние обозначается как инфаркт миокарда без подъёма сегмента ST[14,15].

Нестабильная стенокардия и ИМ без повышения ST являются крайне близкими состояниями, имея общий патогенез и схожую клиническую картину, и могут отличаться только тяжестью симптомов. Для быстрого разграничения внутри ОКС требуется определение уровня соответствующих кардиоспецифических биомаркеров в крови пациента, обладающего наивысшей специфичностью и чувствительностью в отношении некроза миокарда, причём уже в течение короткого времени после начала симптомов ИМ. Современная диагностика инфаркта миокарда должна обязательно включать определение специфических маркеров повреждения миокарда, а именно - кардиотропонинов, СК-МВ и миоглобина. Наиболее информативным является определение количества кардиотропонинов в период 6-24 часов после приступа стенокардии. Результаты исследования могут быть использованы для определения рисков летального исхода, выбора оптимальной тактики ведения больных ИБС, эффективности проводимой терапии. [16,17]

На основании данных многочисленных клинических исследований, в мировой практике выработана определенная тактика лечения пациентов с ОКС с подъемом сегмента ST и без подъема ST и направлена она на восстановление кровотока различными методами, в зависимости от степени повреждения миокарда $[9,10,11]$. За последние годы, в связи с увеличением количества учреждений, в которых работают рентгеноперационные, отмечается тенденция к увеличению числа чрескожных вмешательств у больных с ОКС [12]. Однако, также отмечается и рост числа пациентов с ОКС, которым выполняют хирургическое лечение - коронарное шунтирование (КШ), что обусловлено невозможностью у данных пациентов выполнения эндоваскулярного лечения [12].

\section{Выбор инвазивного метола лечения ОКС}

К инвазивным методам лечения ОКС относят операции шунтирования коронарных артерий и чрескожные коронарные вмешательства. Тенденции в области ак- 
тивного лечения ОКС, согласно актуальным рекомендациям последних десятилетий, смещены в сторону эндоваскулярных процедур. Однако, в целом ряде случаев наиболее эффективным пособием является именно операции аортокоронарного шунтирования (АКШ) [13]. При этом, эндоваскулярное вмешательство часто рассматривается как один из этапов лечения ОКС в авторитетных работах $[13,14]$. Так, в среднем до $30 \%$ больных с ОКС нуждаются в АКШ в отдалённом периоде, вследствие неадекватности экстренной эндоваскулярной реваскуляризации. Это может быть связано с различными факторами, и, в первую очередь, с многочисленными анатомическими особенностями поражений коронарных артерий [14]. Таким образом, при выборе тактики лечения пациента с ОКС необходимо учитывать возможные противоречия: с одной стороны - открытая операция в ранние сроки после инфаркта миокарда часто ассоциируется с высоким уровнем осложнений, с другой - своевременность вмешательства и адекватность выполненной полной реваскуляризации миокарда являются основополагающими факторами стабилизации коронарного кровообращения и предотвращением повторных вмешательств на сердце.

Больным с поражением одного или двух сосудов, как правило, проводится чрескожное вмешательство, с установкой стента на фоне введения блокаторов гликопротеиновых IIb/IIla рецепторов. Аортокоронарное вмешательство у таких больных целесообразно, если анатомия коронарных артерий (выраженная извитость) не позволяет безопасно выполнить чрескожную процедуру. Больным с поражением ствола ЛКА или 3-сосудистым поражением, особенно при нарушенной функции Лж, показано аортокоронарное шунтирование. У больных с двухсосудистым или трёхсосудистым поражением, со стенозом, подходящим для имплантации стентов, показания оценивают индивидуально [15].

Таким образом, при выработке стратегии лечения, применимой для большинства больных, госпитализированных с подозрением на ОКС, в каждом конкретном случае допустимы отклонения от рекомендаций, в зависимости от индивидуальных особенностей больного. Кардиохирург принимает окончательное решение по тактике лечение больного, с учетом анамнеза, клинических симптомов, данных, полученных в ходе наблюдения за больным и обследований за время госпитализации, а также, несомненно, исходя из возможностей лечебного учреждения и собственного опыта.

\section{Хирургическая \\ реваскуляризашия миокарАа}

На сегодняшний день к хирургическим методам лечения ОКС относятся: коронарное шунтирование в условиях ИК, в сочетании с различными вариантами защиты миокарда, в том числе с помощью различных методов кардиоплегии; АКШ на работающем сердце т.н. методика «оff-pump», и коронарное шунтирование в условиях ИК на параллельной перфузии.

\section{1. Коронарное шунтирование в условиях ИК и кардиоплегии}

Кардиохирургами изучался вопрос выбора оптимальной стратегии реваскуляризации для пациентов с ОКС без подъема сегмента ST при многососудистом поражением коронарного русла. В исследование вошли 362 пациента с ОКС. Пациентам выполняли: поэтапное чрескожное вмешательство - группа 1 (было выполнено 266 (73,4\%) пациентам), коронарное шунтирование - группа 2 (было выполнено 72 больным $(19,9 \%)$ больным), первый этап ЧКВ и второй этап КШ группа 3 (реализовано 24 (6,6\%) пациентам). Конечными точками исследования стали такие неблагоприятные кардиоваскулярные события как смерть, инфаркт миокарда (ИМ), острое нарушение мозгового кровообращения/транзиторная ишемическая атака, повторная реваскуляризация целевого сосуда, кровотечение по шкале BARC (Bleeding Academic Research Consortium) [18]. Максимальные значения шкалы GRACE были отмечены в группах 1 и 3, тогда как наибольшая выраженность коронарного атеросклероза по шкале SYNTAX в группах 2 и 3. Показатель летальности колебался от 2,8\% в группе КШ до $8,3 \%$ в группе 1. Частота ИМ от 1,4\% в группе КШ до 8,3\% в группе 3. Встречаемость геморрагических осложнений 3-5 типа по шкале BARC составила от 1,1\% в группе 1 до 37,5\% в группе 3. Таким образом, авторы считают, что отсутствие четких рекомендаций для выбора оптимальной стратегии и сроков реваскуляризации среди пациентов с ОКС без подъёма сегмента ST при многососудистом поражением коронарного русла приводит к развитию значительного числа неблагоприятных кардиоваскулярных исходов в данной группе пациентов. Полученные в исследовании результаты говорят о том, что стратегия реваскуляризации для пациентов с ОКС без подъёма сегмента ST должна осуществляться на основе комплексного применения стратификационных шкал, таких как GRACE, SYNTAX, EuroScore II. При этом, АКШ, как самодостаточный метод, не требующий дальнейших вмешательств, может являться оптимальной стратегией реваскуляризации для значительно большего числа пациентов, чем применяется в настоящее время [19].

Ибрагимов Р.М.О. (2013) в своей работе указывает, что при мультифокальном атеросклерозе, как принципы выбора оптимальной хирургической тактики, так и используемые методы имеют существенные различия между стабильными формами ИБС и ОКС без подъёма 
сегмента ST. Среди пациентов с ОКС с подъёмом сегмента ST, даже при неосложненной его форме, сохраняется высокий риск ранних кардиальных осложнений, что требует достоверно большего числа экстренных и срочных вмешательств, а также необходимости реваскуляризации миокарда первым этапом. Выбор метода коронарного шунтирования преимущественно зависит от клинической формы ИБС: при ОКС без подъёма сегмента ST он достоверно чаще решается в пользу чрескожных вмешательств, при стабильной ИБС - в пользу операций на открытом сердце ( $<<0,0001)$. При этом, по сравнению с первичным коронарным стентированием, АКШ обеспечивает более полный объем восстановления коронарного кровотока ( $p=0,008)$. Автор пишет, что у пациентов с мультифокальным атеросклерозом наиболее оправданным представляется поэтапное лечение, даже в пределах одного сосудистого бассейна. Причем, с наличием ОКС связана необходимость большего количества этапов как на коронарных артериях $(p<0,0001)$, так и общего количества этапов до завершения лечения в полном объеме ( $p<0,0001)$. При этом, коронарное шунтирование и коронарное стентирование рассматриваются автором как высокоэффективные методы реваскуляризации миокарда у больных с мультифокальным атеросклерозом при ОКС без подъёма сегмента ST с низким уровнем тяжелых сердечно-сосудистых осложнений как в ранние, так и в отдаленные сроки наблюдения. Автор делает вывод, что при оптимальном выборе лечебной стратегии количество и структура негативных клинических исходов не зависят от метода реваскуляризации миокарда [3].

Бранд Я.Б., с соавторами (2017) изучали вопрос отдаленных результатов коронарного шунтирования у пациентов с острым коронарным синдромом. Был выполнен анализ отдаленных результатов операции коронарного шунтирования у пациентов с ОКС. В исследование вошло 489 пациентов с ОКС. Объем и сроки хирургического лечения зависели от тяжести состояния пациента и эффективности консервативной терапии, и его выполняли в экстренно или экстренно-отсроченном порядке. В зависимости от начала болевого синдрома в экстренном порядке были прооперированы (в сроки менее 24 ч) 176 пациентов (35,9\%), в экстренно-отсроченном периоде (в сроки от 24 до 48 ч) - 313 пациентов $(64,1 \%)$. По данным большинства международных исследований, выживаемость больных в отдаленном периоде после хирургической реваскуляризации миокарда составляет 75-85\% за 10-летний период наблюдений. Учитывая высокую летальность у больных с ОКС при естественном течении, реваскуляризацию миокарда следует выполнять в кратчайшие сроки. В исследовании пациентов, оперированных в экстренном порядке (в сроки менее 24 ч), было 176 (35,5\%), в экстренно-отсроченном периоде (в сроки от 24 до 48 ч) -
313 (64,4\%). Рецидивы стенокардии были выявлены у 5 пациентов (3,4\%), оперированных в экстренном порядке и у 19 пациентов (6,0\%), оперированных в экстренно-отсроченном периоде. Повторные хирургические вмешательства не выполняли. Двенадцатилетняя выживаемость была прослежена у 56 пациентов $(11,4 \%)$ и составила $98 \%$ [2].

\section{2. Коронарное шунтирование на работающем сердце по методике оff-pump (OPCAB).}

Применение шунтирования коронарных артерий на работающем сердце связано с желанием избежать осложнений, связанных с использованием аппарата ИК. Искусственное кровообращение является причиной системного воспалительного ответа. Существующие стратегии предупреждения постперфузионного системного воспаления не являются достаточно эффективными, поэтому в ряде случаев, когда это возможно, применяется отказ от искусственного кровообращения. Однако, всё ещё многие хирурги относятся к этой методике с опасением, особенно при лечении ОКС. Это связано с тем, что такое шунтирование может закончиться неполной реваскуляризацией, а значит, может потребовать повторных вмешательств, ухудшающих прогноз и качество жизни пациентов. Последние данные свидетельствуют о прогностическом преимуществе традиционного метода АКШ в условиях ИК, однако механизм этого остается неясным. Возможно более низкие результаты при АКШ в отсутствии ИК могут быть обусловлены необходимостью повторной реваскуляризации, причем эта польза становится значимой только при длительном этапе наблюдения[16,19].

Neumann A. и соавторы (2019) изучали целесообразность, эффективность и исходы экстренного коронарного шунтирования в условиях ОКС. Авторами был проведён ретроспективный обзор пациентов с ОКС, оперированных в условиях экстренной хирургии, а также в отсроченном порядке, либо по методике CABG, либо ОРСАВ. В общей сложности в исследование вошло 205 пациентов с ОКС. АКШ с использованием аппарата ИК перенесли 109 пациентов (53,2\%). Операцию ОРСАВ провели 96 пациентам (46,8\%). Уровни

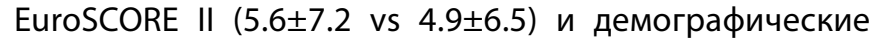
показатели были сопоставимы между группами. Тенденция к снижению послеоперационной летальности наблюдалась у пациентов в группе ОРСАВ (2.1 против 5.5\%). Частота послеоперационного инсульта и синдрома низкого сердечного выброса, а также длительность инотропной поддержки и необходимость повторной стернотомии были значительно ниже в группе ОРСАВ. АКШ, выполненное мгновенно в экстренной ситуации, не было связано с повышением смертности или заболеваемости по сравнению с отсроченными процедура- 
ми. По данным приведённого исследования, операция на работающем сердце у экстренных пациентов была связана с более низкой послеоперационной заболеваемостью и более коротким пребыванием в отделении интенсивной терапии. Различий в полноте реваскуляризации миокарда между группами не наблюдалось, даже в случае экстренных операций. Авторы считают, что операция ОРСАВ безопасна и эффективна при ОКС и может рассматриваться у гемодинамически стабильных пациентов в экстренных ситуациях [13].

Thakur U. и соавторы (2018) считают, что операции АКШ на работающем сердце по методике CABG технически сложнее и теоретически могут привести к менее полной реваскуляризации миокарда. Авторами было проанализировано 13 исследований, включающих в общей сложности 13234 пациента. В исследовании оценивались краткосрочные, среднесрочные и долгосрочные результаты у пациентов, подвергшихся реваскуляризации на работающем сердце и в условиях ИК. Клинические результаты оценивались через 30 дней, через 12 месяцев или через 4 и более лет. Первичным исходом была долговременная смерть от всех причин, в то время как вторичные исходы включали 30-дневную, 12-месячную и более 4-летнюю сердечную смерть, инсульт, инфаркт миокарда или реваскуляризацию. Шунтирование на работающем сердце было связано с повышенным риском смерти от всех причин (отношение шансов (OR) 1,18, 95\% доверительный интервал (CI) 1,02-1,32,) и повторным шунтированием (или 2,57, 95\% Cl 1,23-5,39,) при длительном наблюдении. При наблюдении в течение 12 месяцев после АКШ на работающем сердце было выявлено увеличение необходимости повторных реваскуляризаций (или 1.59, 95\% Cl 1.09-2.33, $\mathrm{p}=0.02$ ). Не было отмечено различий между группами в сроки 30 дней, 12 месяцев и более 4 лет по частоте инфаркта миокарда или инсульта. Таким образом, АКШ на работающем сердце ассоциируется со значительно более высокими показателями смертности при долгосрочном наблюдении. Авторы считают, что данные результаты демонстрируют временную зависимость, за которой могут следовать плохие долгосрочные ре- зультаты в виде более высокой необходимости повторной реваскуляризации в течение одного- или пятилетнего периода наблюдения [18].

Parmeshwar N., и соавторы (2019) сравнивали долгосрочные результаты при операциях на работающем сердце и при использовании аппарата ИК. Всего было проанализировано 555 пациентов, из них коронарное шунтирование с ИК было проведено 238, и АКШ на работающем сердце - 317 больным. Оценивались показатели смертности, наличие нового инфаркта миокарда и необходимость в повторном вмешательстве в период от 8 до 12 лет после первичной операции. Данные настоящего исследования не показали различий в ключевых отдаленных результатах у пациентов, перенесших реваскуляризацию с или без ИК, подтверждая идею о том, что оба метода имеют аналогичные поздние результаты в отношении общей выживаемости, необходимости повторного вмешательства и послеоперационного инфаркта миокарда [15].

Мерзляков В.Ю. и соавторы предлагает использование у больных со сниженной фракцией выброса применение операции прямой реваскуляризации миокарда на работающем сердце как один из методов оперативного лечения ишемической болезни сердца. В статье представлен клинический случай положительного результата лечения больного с ОКС и низкой сократительной способностью миокарда с помощью АКШ на работающем сердце [4].

Исходя из этого, по количеству работ и обсуждаемых в них тем, можно заключить, что проблема коронарного шунтирования на фоне ОКС крайне актуальна в настоящее время. При этом, приоритетное использование того или иного вида хирургического или интервенционного вмешательства должно быть в чёткой зависимости от особенностей конкретного пациента и строго индивидуально. Основные работы по ОКС сходятся в одном главном выводе, что независимо от метода лечения пациента, необходимо придерживаться тактики полной реваскуляризации миокарда.

\section{ЛИТЕРАТУРА}

1. Бокерия Л.А., Голухова Е.З., Сигаев И.Ю., Керен М.А. Современные подходы к хирургическому лечению ишемической болезни сердца у больных сахарным диабетом. Вестник Российской академии медицинских наук. 2012; 67(1):20-26.

2. Бранд Я.Б., Мазанов М.Х., Тимербаев А.В., Сагиров М.А., Харитонова Н.И., Чумаков М.В. Отдаленные результаты коронарного шунтирования у пациентов с острым коронарным синдромом. ГБУЗ «НИИ скорой помощи им. Н.В. Склифосовского Департамента здравоохранения г. Москвы», Москва, Российская Федерация. 2017 DOI: 10.23934/2223-9022-2017-6-3-251-256

3. Ибрагимов Р.М.О. Результаты прямой реваскуляризации миокарда при ОКС без стойкого подъема сегмента ST у больных мультифокальным атеросклерозом тема диссертации и автореферата по ВАК РФ 14.01.26, кандидат медицинских наук.

4. Мерзляков В.Ю., Скопин А.И., Мамедова С.К., Абаджян М.Ф., Гатамова Н.А. Аортокоронарное шунтирование по жизненным показаниям у больного с низкой сократительной способностью миокарда. Номер: 2 Год: 2013 Страницы: 32-35. 
5. Мовсесян Р.А., Алшибая М.М., Коваленко О.А., Работников Ю.В. Хирургическое лечение острого коронарного синдрома на современном этапе Бюллетень НЦССХ им. А.Н. Бакулева РАМН Сердечно-сосудистые забьолевания. Издательство: Национальный медицинский исследовательский центр сердечно-сосудистой хирургии имени А.Н. Бакулева (Москва) Том: 9 Номер: S6 Год: 2008 C. 80 ISSN: 1810-0694.

6. Сулимов В.А. Острый коронарный синдром без подъёма сегмента ST современные возможности диагностики и лечения. АтмосферА. Кардиология № 3.2005.

7. Эрлих А.Д. Регистры острых коронарных синдромов — их виды, характеристики и место в клинической практике 2012.

8. Hlatky M.A., Boothroyd D.B., Melsop K.A., et al. Medical costs and quality of life 10 to 12 years after randomisation to angioplasty or bypass surgery for multivessel coronary artery disease. Circulation. 2004; 110(14): 1960-1966. PMID: 15451795.

9. Libby P. Mechanisms of acute coronary syndromes and their implications for therapy. N Engl J Med. 2013; 368(21): 2004-2013. PMID: 23697515. D0I: 10.1056/ NEJMra1216063.

10. Management of acute coronary syndromes without persistent ST segment elevation. Recommendation of task force of European Society of Cardiology. Eur Heart J 2000;21:1406-1432.].

11. Murray CJ, Lopez AD. Alternative projections of mortality and disability by cause 1990-2020: Global Burden of Disease Study. Lancet 1997;349:1498-1504

12. McManus D.D., Gore J., Yarzebski J., et al. Recent trends in the incidence, treatment, and outcomes of patients with STEMI and NSTEMI. Am J Med. 2011; 124: 40-47. PMID: 21187184. D0I: 10.1016/j.amjmed.2010.07.023.

13. Neumann A., Vöhringer L., Fischer J., et al. Off-Pump Coronary Artery Bypass Grafting in Acute Coronary Syndrome: Focus on Safety and Completeness of Revascularization. Thorac Cardiovasc Surg. 2019 Feb 6. doi: 10.1055/s-0039-1677834.

14. Paez R.P., Hossne Junior N.A., Santo JADE et al. BYPASS Registry Study Group Coronary Artery Bypass Surgery in Brazil: Analysis of the National Reality Through the BYPASS Registry. Braz J Cardiovasc Surg. 2019 Mar-Apr;34(2):142-148.

15. Parmeshwar N., Fero K.E., Manecke G., Coletta J.M. Off-Pump Versus On-Pump: Long-Term Outcomes After Coronary Artery Bypass in a Veteran Population. J Cardiothorac Vasc Anesth. 2019 May;33(5):1187-1194. doi: 10.1053/j.jvca.2018.11.003. Epub 2018 Nov 3.

16. Simoons M.L. Cardiovascular disease in Europe: challenges for the medical profession. Opening address of the 2002 Congress European Society of Cardiology (editorial). Eur. Heart J. 2003; 24: 8-12.

17. Terkelsen C.J., Lassen J.F., Norgaard B.L., et al. Mortality rates in patients with ST-elevation vs. non-ST-elevation acute myocardial infarction: observations from an unselected cohort. Eur Hear

18. Thakur U., Nerlekar N., Muthalaly R.G., et al. Off- vs. On-Pump Coronary Artery Bypass Grafting Long-Term Survival is Driven by Incompleteness of Revascularisation. Heart Lung Circ. 2018 Dec 13. pii: S1443-9506(18)32006-7. doi: 10.1016/j.hlc.2018.11.019.

19. Uretsky B.F., Farcuhar D.S., Boresin A., Hood W.E. Symptomatic myocardial infarction without chest pain; prevalens and clinical course. Am J Cardiol 1977;40:498503. Logon R., Wong F., Barclay J. Symptoms associated with myocardial infarction: arc they of diagnostic value? N Z Med J 1986;99:276-278. 